\title{
ON THE A.C. SUSCEPTIBILITY OF METALS IN THE NORMAL OR SUPERCONDUCTING STATE
}

\author{
L. J. M. VAN DE KLUNDERT, E. A. GIJSBERTSE and L. C. VAN DER MAREL \\ Afdeling der Technische Natuurkunde van de Technische Hogeschool Twente, \\ Enschede, Nederland
}

Received 3 Mei 1973

\section{Synopsis}

Models describing the a.c. behaviour due to surface superconductivity can be extended to include frequency effects. It is necessary to take normal currents into account and to assume that superconducting currents can only change under the influence of electrical fields. The model has been verified by means of susceptibility measurements and direct observation of the local $b-h$ loops.

1. Introduction. Many processes involving the magnetic behaviour of nonconducting materials can be described in terms of one or more relaxation times. A similar description method is used for the behaviour of (non-magnetic) conductors subject to time-dependent fields. Several techniques have been developed to investigate the values of these time constants. Step-field methods have been used, for example, for paramagnetic salts when the relaxation time is larger than one second ${ }^{1}$ ). In conductivity measurements the step-field response method is applied for $\tau$-values down to a few microseconds ${ }^{2,3,4}$ ).

Another method, introduced in the thirties in the field of paramagnetism of non-conducting magnetic materials is the induction $\operatorname{method}^{5}$ ). Materials are here investigated in magnetic fields: $H=H_{0}+h_{0} \mathrm{e}^{-\mathrm{i} \omega t}, h_{0} / / H_{0}$ and $\left|h_{0}\right| \ll\left|H_{0}\right|$. These induction measurements, overshadowed now by resonance experiments, largely contributed to the insight in paramagnetic relaxation processes. They gave the impetus to the famous papers of Van Vleck ${ }^{6}$ ) in 1941 where quantitative calculations about temperature and magnetic-field behaviour of relaxation times were presented and where for the first time the existence of a phonon bottleneck was argued. Results of measurements on magnetic salts of different concentration of the magnetic ions for the second time convinced Gorter et al. ${ }^{7}$ ) of the existence of this phonon bottleneck and in the same school of Gorter it was demonstrated that also the heat conductivity of the crystal as well as the Kapitza resistance between sample and liquid helium may influence the relaxation processes ${ }^{8}$ ). 
Originally these induction methods were developed with experiments on metals. Already in 1902 Zenneck $^{9}$ ) applied such a method on non-magnetic metals and in 1919 Wwedensky $^{10}$ ) investigated magnetic metals with the same method. Later on Kouwenhoven and Daiger ${ }^{11}$ ) succeeded in extending largely the frequency range of the self-inductance bridge and in 1955 for the first time a Hartshorn-bridge device was used in the Leiden laboratory ${ }^{12}$ ). With an analogous method we performed in our laboratory a number of experiments on some metals. The response of the coil system which surrounds the cylindrical samples contains the information on the conductive and magnetic properties of the metal to be investigated. When the sample is a non-magnetic normal conducting solid cylinder the response is linear with respect to the amplitude of the modulating field and depends only on the frequency and the conducting properties. In the case of samples in the superconducting state the response will be in general nonlinear and depends strongly on frequency, amplitude and pinning mechanisms.

The basic features of the theory of the a.c. behaviour were outlined in the early sixties by introducing the concept of the critical state ${ }^{13,14}$ ). After that many refinements concerning the field dependence of the critical current and the relation to the pinning mechanisms were published ${ }^{15,16}$ ). Since in the critical-state model surface currents and flux gradients play an important rôle, thin-walled cylinders have great advantage in showing the effects of the associated currents in an enhanced way.

In this paper we shall confine ourselves to the influence of geometrical factors on the a.c. behaviour of metals both in the normal and superconducting states. In section 2 the general solution method of normal conducting hollow and solid cylinders is outlined and the obtained results are presented. An extension to superconducting materials in the vicinity of $T_{c}$ is given. In section 3 the well-known Fink-Rollins-Silcox ${ }^{17,18}$ ) model is extended to include frequency-dependent effects. Comparison with our experimental results is made in section 4 and leads to further extensions of the prior model which are presented in section 5 .

2. Influence of the geometry on the a.c. susceptibility. The susceptibility $\chi$ of a normal conducting cylinder is defined as

$$
\chi=-1+\mu=-1+2 \int_{0}^{a} B(r) r \mathrm{~d} r / a^{2} B(a)
$$

where $B(r)$ is the complex amplitude of the induction inside the cylinder at a distance $r$ from the axis and $B(a)$ the amplitude of the applied field at the sample surface $(r=a)$. Values of $\chi$ can be calculated starting from Maxwell's equations:

$$
\begin{aligned}
& \nabla \times E=\mathrm{i} \omega B, \\
& \nabla \times H=j
\end{aligned}
$$


Introducing appropriate constitutive equations governing the magnetic and conductive properties, e.g.,

$$
\boldsymbol{B}=\mu_{0} \boldsymbol{H}, \boldsymbol{j}=\boldsymbol{\sigma} E
$$

(factors $\mathrm{e}^{-\mathrm{i} \omega t}$ are omitted for shortness; terms involving $\dot{D}$ can be shown to be negligible at the low frequencies here of interest). Introduction of cylindrical coordinates with $z$ parallel to the cylinder axis and the applied field $\boldsymbol{H}=\left(0,0, H_{z}\right)$ yields the following form of Maxwell's equations:

$$
E_{\theta} / r+\partial E_{\theta} / \partial r=\mathrm{i} \omega B_{z}, \quad-\partial H_{z} / \partial r=j_{\theta} .
$$

Inserting the constitutive equations we obtain the following differential equations for $H_{z}$ and $E_{\theta}$ :

$$
\begin{aligned}
& (k r)^{2} \partial^{2} E_{\theta} / \partial(k r)^{2}+k r \partial E_{\theta} / \partial(k r)+\left\{(k r)^{2}-1\right\} E_{\theta}=0 \\
& (k r)^{2} \partial^{2} B_{z} / \partial(k r)^{2}+k r \partial B_{z} / \partial(k r)+(k r)^{2} B_{z}=0
\end{aligned}
$$

with $k^{2}=i \omega \mu_{0} \sigma$. The general solution of (2.6) is

$$
\begin{aligned}
& B_{z}(r)=\alpha_{1} J_{0}(k r)+\alpha_{2} Y_{0}(k r), \\
& E_{\theta}(r)=\beta_{1} J_{1}(k r)+\beta_{2} Y_{1}(k r),
\end{aligned}
$$

$J_{n}$ and $Y_{n}$ being the Bessel and Neumann function of order $n$, respectively. The boundary conditions on the solution for $B_{z}$ can be inserted by noticing that $B_{z}$ should be bounded everywhere in the sample and continuous across the sample surface $r=a$. So we obtain

$$
\begin{aligned}
& x_{1}=B_{z}(a) / J_{0}(k a), \quad \alpha_{2}=0, \\
& \beta_{1}=\mathrm{i} \omega B_{z}(a) / k J_{0}(k a), \quad \beta_{2}=0 .
\end{aligned}
$$

Applying the definition of susceptibility (2.1) leads to

$$
\chi=J_{2}(k a) / J_{0}(k a)=-1+2 J_{1}(k a) / k a J_{0}(k a) .
$$

Formally, the complex skin depth $\delta=\mathrm{i} / k$ can be assumed to depend on such parameters as frequency, external fields, conductive properties, i.e., $\delta=$ $\delta\left(\omega, H_{0}, \sigma, \ldots\right)$. So if we put $k a=z=r \mathrm{e}^{\mathrm{i} \phi}, r$ and $\phi$ both may depend on these parameters:

$$
\begin{aligned}
r & =r\left(\omega, H_{0}, \sigma, \ldots\right), \\
\phi & =\phi\left(\omega, H_{0}, \sigma, \ldots\right) .
\end{aligned}
$$

Although it may not be possible to calculate the functions (2.9a and b) explicitly, it will be useful to express experimental results in terms of $r$ and $\phi$ rather than $\chi^{\prime}$ and $\chi^{\prime \prime}$. This is always possible since (2.8) defines a one to one correspondence from the $\chi^{\prime}-\chi^{\prime \prime}$ plane to the $r-\phi$ plane $(0 \leqq \phi \leqq 2 \pi, 0 \leqq r<\infty)$. In fig. 1 this is illustrated by drawing lines of constant $r$ and $\phi$ in the $\chi^{\prime}-\chi^{\prime \prime}$ plane. The lines 


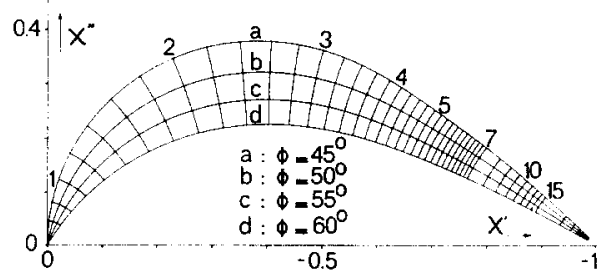

Fig. 1. Lines of constant $r$ and $\phi$ in the $\chi^{\prime}-\chi^{\prime \prime}$ plane for a solid cylinder (2.8). Values of $r$ are indicated.

$\phi=\pi / 4$ and $\phi=\pi / 3$ denote the classical skin effect and the anomalous limit, respectively. It may be mentioned here that for a slab of thickness $2 d$ an analogous procedure leads to the result:

$$
\chi=-1+\operatorname{tg}(k d) / k d .
$$

In practice the shape of the $\chi^{\prime}-\chi^{\prime \prime}$ curves is not much influenced by the geometry of the sample as can be seen from fig. 2 .

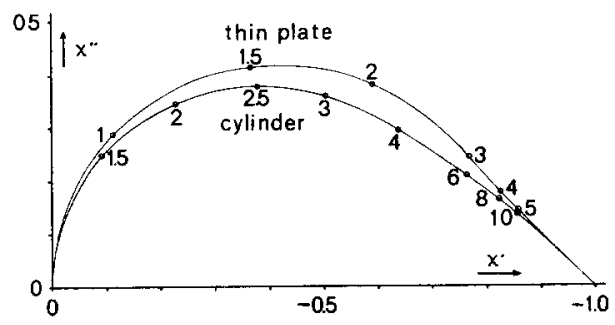

Fig. 2. Comparison of the $\chi^{\prime}-\chi^{\prime \prime}$ curves for a solid cylinder (2.8) and a thin plate (2.10). Dimensionless values of $r$ are indicated on the curves; $r=k a \mathrm{e}^{-\pi \mathrm{i} / 4}$ for a cylinder and $k d \mathrm{e}^{-\pi \mathrm{i} / 4}$ for a thin plate.

As an extension of the former calculation we shall treat the case of the hollow cylinder with inner radius $b$ and outer radius $a$. Using appropriate boundary conditions we arrive at the result

$$
\chi=-1+\frac{2}{k a} \frac{J_{1}(k a)-\alpha Y_{1}(k a)}{J_{0}(k a)-\alpha Y_{1}(k a)},
$$

with

$$
\alpha=\frac{k b J_{0}(k b)-2 J_{1}(k b)}{k b Y_{0}(k b)-2 Y_{1}(k b)} .
$$

In fig. 3a the $\chi$ dependence on the ratio $b / a$ is shown for some fixed normalized frequencies. The frequency behaviour is shown in fig. $3 b$ for some fixed values of $b / a$. It is noted that in the limit $b \rightarrow a$ the $\chi^{\prime}-\chi^{\prime \prime}$ curve tends to a half circle which can be interpreted as the diamagnetic analogue of the Casimir-Du Pré ${ }^{19}$ ) curves 


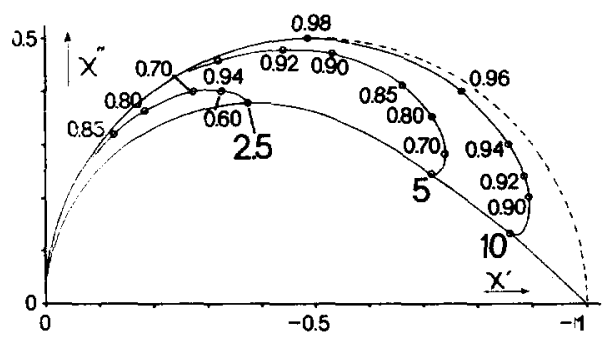

a

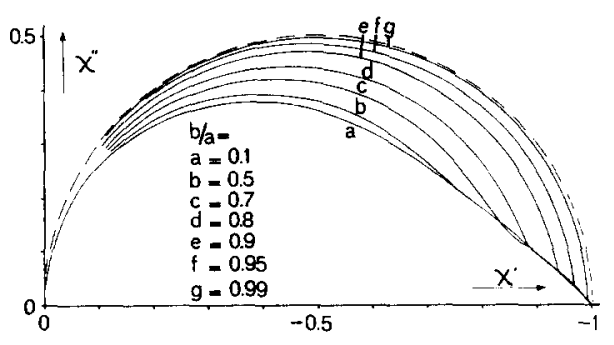

b

Fig. 3a. $\chi$ values for hollow cylinders (2.11) for some $r$ values; $r=k a \mathrm{e}^{-\pi \mathrm{i} / 4}=2.5,5$ and 10 . The figures along the lines indicate $b / a$ values.

Fig. 3 b. $\chi$ values for hollow cylinders (2.11) for some $b / a$ values. The parameter along each curve is $r(0<r<\infty)$.

for non-conducting paramagnetic materials. Formula (2.11) is also valid for the case of concentric solid cylinders with different conductivities $\sigma_{2}$ and $\sigma_{1}$ in the regions $0 \leqq r \leqq b$ and $b \leqq r \leqq a$, respectively. The value of $\alpha$ is then given by

$$
x=\frac{k_{2} J_{1}\left(k_{1} b\right) J_{0}\left(k_{2} b\right)-k_{1} J_{0}\left(k_{1} b\right) J_{1}\left(k_{2} b\right)}{k_{2} Y_{1}\left(k_{1} b\right) J_{0}\left(k_{2} b\right)-k_{1} Y_{0}\left(k_{1} b\right) J_{1}\left(k_{2} b\right)},
$$

with $k_{1}{ }^{2}=\mathrm{i} \omega \mu_{0} \sigma_{1}$ and $k_{2}{ }^{2}=\mathrm{i} \omega \mu_{0} \sigma_{2}$. Without derivation ${ }^{20}$ ) we may mention the more general formulae of $\chi$ for an arbitrary $j(E)$ relation:

$$
\begin{aligned}
& \chi=\mu_{0} \int_{0}^{a} r^{2} j(r) \mathrm{d} r / a^{2} B_{z}(a), \\
& \chi=\left\{E(a)-a E^{\prime}(a)\right\} /\left\{E(a)+a E^{\prime}(a)\right\},
\end{aligned}
$$

with $E^{\prime}(a)=\left\{\mathrm{d} E_{\theta}(r) / \mathrm{d} r\right\}_{r=a}$.

Especially the latter is useful in anomalous skin-effect calculations where the relation $\boldsymbol{j}(\boldsymbol{E})$ is rather complicated ${ }^{21}$ ).

A further extension of this formulation may be obtained for the low-frequency behaviour of superconductors in the Meissner state (see e.g. Kubo ${ }^{22}$ )). Calculation of $\chi$ now results in the same relation (2.8) but with $k^{2}=k_{\mathrm{s}}^{2}=\mathrm{i} \omega \mu_{0} \sigma$ ( $\left.1-x-x / i \omega \tau_{\mathrm{F}}\right), x=n_{\mathrm{s}} / n$ being the fraction of superconducting electrons; $\tau_{\mathrm{F}}$ is the electron relaxation time at the Fermi surface. At temperatures well below $T_{\mathrm{c}}$, $k_{\mathrm{s}}^{2}$ becomes independent of $\omega$ at sufficient low frequencies: $k_{\mathrm{s}}^{2}=-\mu_{0} n_{\mathrm{s}} e^{2} / m$. The real part of the corresponding skin depth equals the London penetration depth $\lambda_{\mathrm{L}}$.

For a more extensive discussion we refer to the thesis of one of us (V.d. K. $)^{20}$ ).

3. Frequency effects in hollow superconducting cylinders. In the literature two mechanisms are proposed to describe the static magnetization curves of superconductors: surface currents and bulk currents. To distinguish between their contributions we performed measurements on thin-walled hollow cylinders and com- 
pared the results with calculations according to some models, proposed in this paper. The Fink-Rollins-Silcox model ${ }^{17,18}$ ) describes the magnetic behaviour of an infinitely long superconducting specimen of arbitrary cross section by stating a local $b-h$ loop as shown in fig. 4 a. The horizontal parts arise from a surface current counteracting the changes in the applied field whereas the tilted parts with slope 1 account for the fact that the supercurrents cannot exceed some maximum value $j_{\max }$. Throughout this paper we shall assume the diamagnetic and paramagnetic screening current density to have the same extreme value $j_{\max }\left(H_{0}\right)$, only depending on the external field. From simple Fourier analysis, the components of $\chi$ can be written (for $\theta_{2}$ see fig. $4 a$ ):

$$
\begin{aligned}
& 1+\chi^{\prime}=\mu^{\prime}=\pi^{-1}\left(\pi-\theta_{2}+\sin \theta_{2} \cos \theta_{2}\right), \\
& \chi^{\prime \prime}=\mu^{\prime \prime}=\pi^{-1} \sin ^{2} \theta_{2} .
\end{aligned}
$$

The main discrepancies between the above outlined one-parameter model and the experimental results on $\chi$ or the observed shape of the $b-h$ loop can be summarized as follows.

a) There is a large influence of the frequency.

b) Observed $b-h$ loops never show a horizontal part, indicating that an electrical field is necessary to change the value of the supercurrent.

c) Measured values of $\left(\partial \chi^{\prime \prime} / \partial \chi^{\prime}\right)_{\chi^{\prime}=-1}$ have a finite value in contradiction to the infinite value of the above model.

The simplest way to include frequency effects is to take into account normal currents. For a thin-walled cylinder, wall thickness $d$, radius $r, d \ll r$, Maxwell's equations are approximated by:

$$
\begin{aligned}
& E=-\frac{1}{2} r \mu_{0} \mathrm{~d} h_{\mathrm{i}} / \mathrm{d} t, \\
& h_{\mathrm{i}}-h=j_{\text {tot }} \mathrm{d},
\end{aligned}
$$

$h_{\mathrm{i}}$ and $h$ being the fields inside and outside the cylinder, respectively. Further we assume

$$
j_{\mathrm{tot}}=j_{\mathrm{n}}+j_{\mathrm{s}}, \quad j_{\mathrm{n}}=\sigma E,
$$

$j_{\mathrm{n}}$ and $j_{\mathrm{s}}$ being the normal and superconducting current densities, respectively. Combining (3.3)-(3.5) we obtain a differential equation for the field $h_{\mathrm{i}}$ :

$$
\tau \mathrm{d} h_{\mathrm{i}} / \mathrm{d} t+h_{\mathrm{i}}=h_{0} \cos \omega t+j_{\mathrm{s}} d,
$$

$\tau=\frac{1}{2} r d \mu_{0} \sigma$ is a time constant depending on geometrical factors as well as material constants.

In (3.6) $j_{\mathrm{s}}$ will vary between the extreme values $\pm j_{\max }$. Let for 


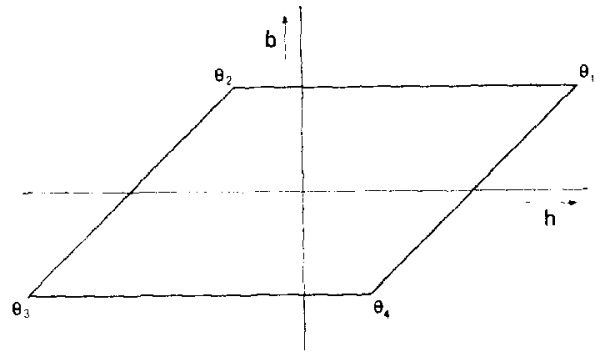

a

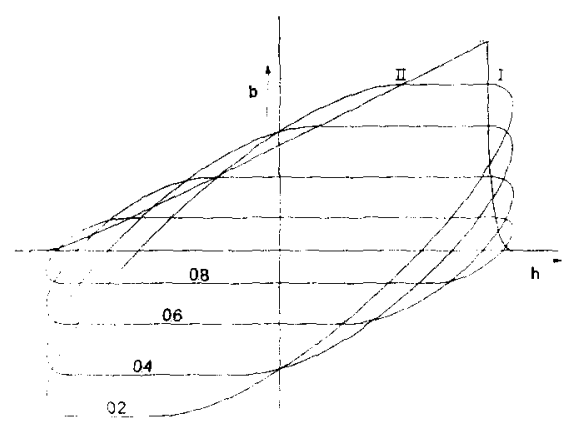

c

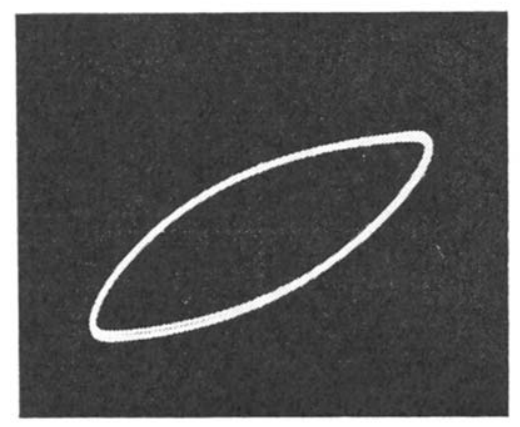

e

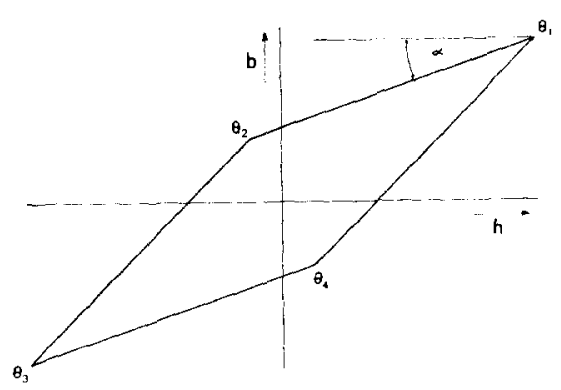

b

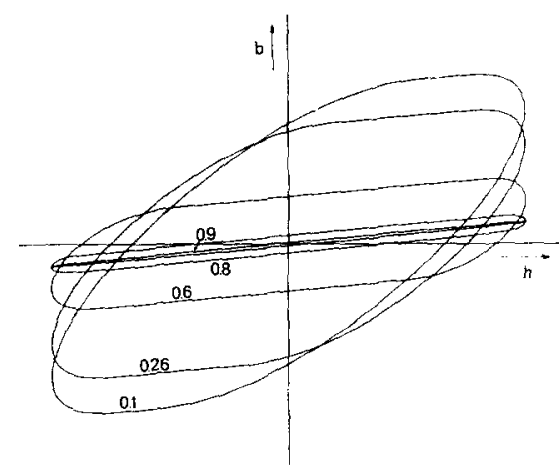

d

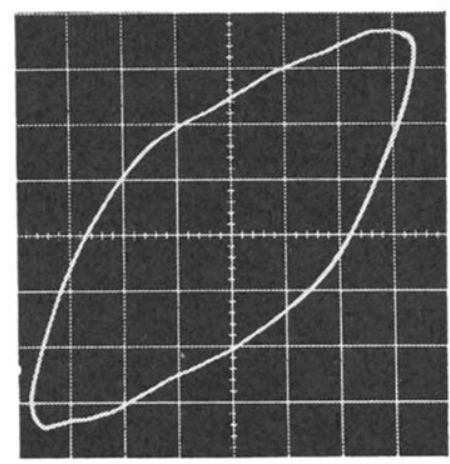

f

Fig. 4a. $b-h$ loop according to Rollins and Silcox ${ }^{18}$ ).

Fig. 4b. Assumed zero-frequency limit $b-h$ loop (see section 5).

Fig. 4c. Calculated $b-h$ loops according to (3.9) and (3.10); $\omega \tau=0.51$. The lines $I$ and $I 1$ indicate the values of $\theta_{1}$ and $\theta_{2}$ depending on $\alpha$. The values of $\alpha$ are indicated in the figure.

Fig. 4d. Calculated $b-h$ loop according to (3.9) and $(5.4) ; \tau / \tau_{1}=10, \omega \tau=0.73$. The values of $\alpha$ are indicated in the figure.

Fig. 4e. Observed $b-h$ loop after heat treatment; $T=4.2 \mathrm{~K}, f=128 \mathrm{~Hz}, h_{0}=11 \mathrm{Oe}, H_{0}=$ $1500 \mathrm{Oe}\left(\approx H_{c_{1}}\right)$.

Fig. 4f. Observed $b-h$ loop before heat treatment; $T=4.2 \mathrm{~K}, f=5 \mathrm{~Hz}, h_{0}=31 \mathrm{Oe}, H_{0}=$ $4960 \mathrm{Oe}\left(>H_{c_{2}}\right)$. 


$$
\left.\begin{array}{llll}
\theta_{4} \leqq \theta \leqq \theta_{1}: & j_{\mathrm{s}}=-j_{\max }, & h_{\mathrm{i}} / h_{0}=f(\theta), & E<0 \\
\theta_{1}<\theta<\theta_{2}: & j_{\mathrm{s}} \neq \pm j_{\max }, & h_{\mathrm{j}} / h_{0}=g(\theta), & E \geqq 0 \\
\theta_{2} \leqq \theta \leqq \theta_{3}: & j_{\mathrm{s}}=+j_{\max }, & h_{\mathrm{i}} / h_{0}=-f(\theta-\pi), & E>0 \\
\theta_{3}<\theta<\theta_{4}+2 \pi: & j_{\mathrm{s}} \neq \pm j_{\max }, & h_{\mathrm{i}} / h_{0}=-g(\theta-\pi), & E \leqq 0
\end{array}\right)
$$

Here $\theta_{1}$ and $\theta_{2}$ are determined by the boundary conditions

$$
(\partial f / \mathrm{d} \theta)_{\theta=\theta_{1}}=0, \quad f\left(\theta_{2}-\pi\right)+g\left(\theta_{2}\right)=0, \quad f\left(\theta_{1}\right)=g\left(\theta_{1}\right) .
$$

With the substitutions $\omega \tau=\operatorname{cotg} \phi$ and $j_{\max } d / h_{0}=\alpha$ the solution for $f$ reads:

$$
f=-\alpha+\delta_{1} \mathrm{e}^{-\theta \operatorname{tg} \phi}+\sin \phi \sin (\phi+\theta) .
$$

From the boundary conditions follows $\delta_{1}=\mathrm{e}^{\theta_{1} \operatorname{tg} \phi} \cos \phi \cos \left(\phi+\theta_{1}\right)$. The simplest solution concerning $g$ we obtain by assuming $E=0$, thus

$$
g=-\alpha+\cos \theta_{1}=f\left(\theta_{1}\right) .
$$

The components of $\chi$ are calculated from the corresponding Fourier integrals:

$$
\begin{aligned}
1+\chi^{\prime} & =\mu^{\prime}=\pi^{-1} \sin \phi\left\{\left(\pi-\theta_{2}+\theta_{1}\right) \sin \phi+\sin \left(\theta_{1}-\theta_{2}\right) \sin \left(\theta_{1}+\theta_{2}-\pi\right)\right\} \\
\chi^{\prime \prime} & =\mu^{\prime \prime}=\pi^{-1} \sin \phi\left\{\left(\pi-\theta_{2}+\theta_{1}\right) \cos \phi-\sin \left(\theta_{1}-\theta_{2}\right) \cos \left(\theta_{1}+\theta_{2}-\pi\right)\right\}
\end{aligned}
$$

It may be noted that for $\alpha=0: \theta_{1}=\theta_{2}=\pi / 2-\phi$ and $\mu^{\prime}$ and $\mu^{\prime \prime}$ reduce to values on the half-circle as discussed in the preceding section.

In fig. $4 c$ some $b-h$ loops for typical values of $\omega \tau$ and $\alpha$ are given. In fig. 5 the frequency dependence of the dissipation as function of $\alpha$ is presented.

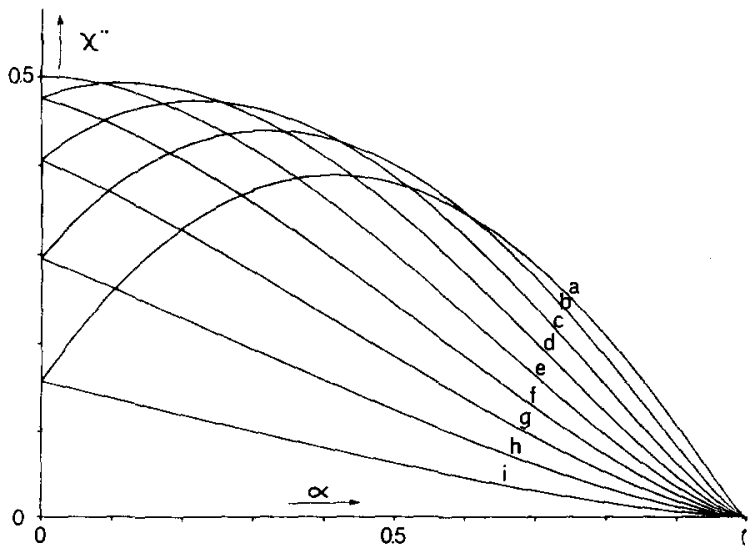

Fig. 5. $\chi^{\prime \prime}$ as a function of $\alpha$ at different frequencies; $\omega \tau=\operatorname{cotg} \phi$ with $\phi=k \pi / 20, k=9,8, . .1$ for the curves $a-i$, respectively. 
4. Experiments. The experiments were performed on a hollow niobium cylinder of $25 \mathrm{~mm}$ length, $3 \mathrm{~mm}$ outer radius and $0.3 \mathrm{~mm}$ wall thickness. Data were obtained before and after the sample had passed an inductive heat treatment at $2200^{\circ} \mathrm{C}$ for 2 hours in a vacuum better than $10^{-5}$ torr.

The consequences of the model in the preceding section were verified by means of two types of experiments. First the a.c. susceptibility was measured with a Hartshorn bridge ${ }^{20}$ ). Secondly the off-balance voltage of the bridge was integrated to show the local $b-h$ loops on an oscilloscope and recorded on $16 \mathrm{~mm}$ film. The three parameters that have been varied are the frequency and amplitude of the modulating field and the static field. Above $H_{c 2}$ no hysteretic behaviour was observed. The frequency dependence of $\chi$ of the sample before the heat treatment is presented in fig. 6 . The calculated frequency dependence of the $\chi^{\prime}-\chi^{\prime \prime}$ curves fits

a

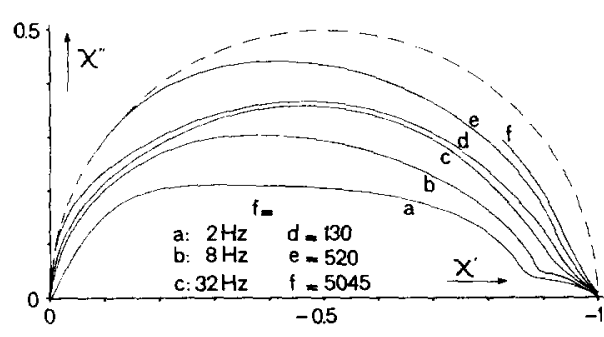

b

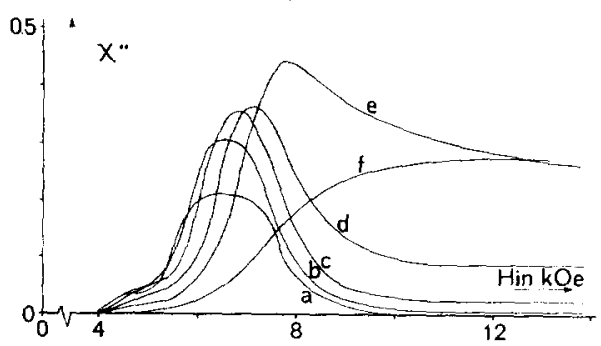

Fig. 6a. $\chi^{\prime}-\chi^{\prime \prime}$ curves at different frequencies; $T=4.2 \mathrm{~K}, h_{0}=6$ Oe. The parameter along each curve is the static field.

Fig. 6b. Corresponding $\chi^{\prime \prime}$ vs. $H_{0}$ curves.

rather well the observed shape at high values of the static field and not too small values of the amplitude. However, the fact that an amplitude dependence of the $\chi^{\prime}-\chi^{\prime \prime}$ curve experimentally is also found cannot be explained with the help of the two-parameter model of section 3. The influence of the amplitude can also be seen by plotting $\chi^{\prime \prime}$ as a function of $\log h_{0}$ at fixed values of $H_{0}$ and comparing it with the function predicted in section 3 . In fig. 7 it is shown that although a single function can describe the $\chi^{\prime \prime}-h_{0}$ dependence (even to values of $H_{0}$ below $H_{c 2}$ after the sample has been heat-treated) this function largely deviates from the predicted shape. The main reason for this discrepancy can be found from the shape

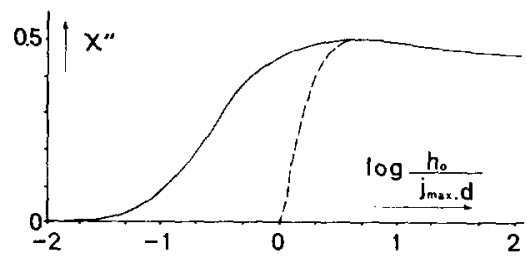

Fig. 7. $\chi^{\prime \prime}$ vs. $\log h_{0} / j_{\max } d$ at $130 \mathrm{~Hz}(\omega \tau \approx 1)$ after heat treatment. Solid line: experimental values. Dashed line: according to (3.11) with $\omega \tau \approx 1$. 
of the $b$ - $h$ loops. Some are presented in fig. $4 \mathrm{e}$ and $\mathrm{f}$. It is seen there that no horizontal parts occur, in contradiction to our assumptions.

5. Extensions of the models of section 3. To overcome the difficulties mentioned before we must supplement the assumptions of section 3 . The absence of horizontal parts indicates that the changes in the superconducting current are caused by an electrical field and can be described by a London-like equation:

$$
\mathrm{d} j_{\mathrm{s}} / \mathrm{d} t=\sigma E / \tau_{1} .
$$

For the zero-frequency limit $b-h$ loop (see fig. $4 b$ ) this means a parallelogram with non-horizontal parts providing an electrical field in accordance with Maxwell's equation (3.3). For this type of $b-h$ loop $\chi^{\prime}$ and $\chi^{\prime \prime}$ can be written:

$$
\left.\begin{array}{rl}
1+\chi^{\prime} & =\mu^{\prime}=\pi^{-1}\left\{\pi-\left(\theta_{2}-\sin \theta_{2} \cos \theta_{2}\right)(1-\operatorname{tg} \alpha)\right\}, \\
\chi^{\prime \prime} & =\mu^{\prime \prime}=\pi^{-1} \sin ^{2} \theta_{2}(1-\operatorname{tg} \alpha),
\end{array}\right\}
$$

(for $\operatorname{tg} \alpha$ and $\theta_{2}$ see fig. $4 \mathrm{~b}$ ).

The extension of the second model of section 3 is rather complicated in its interpretation. We therefore shall confine ourselves to the formulation and solution of the problem and leave the derivation and comparison with experiment to a subsequent paper ${ }^{23}$ ). The solution for $f$ remains unchanged whereas $g$ now obeys (3.6) with

$$
j_{\mathrm{s}}=\left(\sigma / \tau_{1}\right) \int_{t_{1}}^{t} E \mathrm{~d} t-j_{\max }=-\left(\tau / \tau_{1}\right) h_{\mathrm{i}}(t)-j_{\max }+\left(\tau / \tau_{1}\right) h_{i}\left(t_{1}\right) .
$$

For $g$ then follows:

$$
\begin{aligned}
g(\theta)= & -\alpha+\cos \theta_{1}(1-\operatorname{tg} \phi / \operatorname{tg} \psi) \\
& +\operatorname{tg} \phi / \operatorname{tg} \psi\left\{\delta_{2} \mathrm{e}^{-\theta \operatorname{tg} \psi}+\sin \psi \sin (\psi+\theta)\right\} .
\end{aligned}
$$

with $\delta_{2}=\mathrm{e}^{\theta_{1} \operatorname{tg} \psi} \cos \psi \cos \left(\psi+\theta_{1}\right)$ (from $\left.(\mathrm{d} g / \mathrm{d} \theta)_{\theta=\theta_{1}}=0\right) ; \operatorname{tg} \psi=\left(1+\tau / \tau_{1}\right) \operatorname{tg} \phi$. The boundary condition (3.8) now allows for several solutions of $\theta_{1}, \theta_{2}$-combinations of which the following two cases may be mentioned:

$$
\begin{aligned}
& \text { A. } \theta_{2}-\theta_{1}<\pi, \text { i.e., } \pi / 2-\psi<\theta_{1} \leqq \pi / 2-\phi, \\
& 1+\chi^{\prime}=\mu^{\prime}=\pi^{-1} \operatorname{tg} \phi\left[\operatorname { c o s } \phi \left\{\left(\pi-\theta_{2}+\theta_{1}\right) \sin \phi+\sin \left(\theta_{2}-\theta_{1}\right) \sin \left(\theta_{2}+\theta_{1}\right.\right.\right. \\
& +\phi)\}+\cos \psi\left\{\left(\theta_{2}-\theta_{1}\right) \sin \psi-\sin \left(\theta_{2}-\theta_{1}\right) \sin \left(\theta_{2}+\theta_{1}+\psi\right)\right\} \\
& -2 \cos ^{2} \phi \cos \left(\theta_{1}+\phi\right)\left\{\cos \left(\theta_{1}-\phi\right)+\cos \left(\theta_{2}-\phi\right) \mathrm{e}^{\left(\pi-\theta_{2}+\theta_{1}\right) \operatorname{tg} \phi}\right\} \\
& \left.+2 \cos ^{2} \psi \cos \left(\theta_{1}+\psi\right)\left\{\cos \left(\theta_{1}-\psi\right)+\cos \left(\theta_{2}-\psi\right) \mathrm{e}^{\left(\theta_{1}-\theta_{2}\right) \operatorname{tg} \psi}\right\}\right], \\
& \chi^{\prime \prime}=\mu^{\prime \prime}=\pi^{-1} \operatorname{tg} \phi\left[\cos \phi\left\{\left(\pi-\theta_{2}+\theta_{1}\right) \cos \phi-\sin \left(\theta_{2}-\theta_{1}\right) \cos \left(\theta_{2}+\theta_{1}+\phi\right)\right\}\right. \\
& +\cos \psi\left\{\left(\theta_{2}-\theta_{1}\right) \cos \psi+\sin \left(\theta_{2}-\theta_{1}\right) \cos \left(\theta_{2}+\theta_{1}+\psi\right)\right\} \\
& -2 \cos ^{2} \phi \cos \left(\theta_{1}+\phi\right)\left\{\sin \left(\theta_{1}-\phi\right)+\sin \left(\theta_{2}-\phi\right) \mathrm{e}^{\left(\pi-\theta_{2}+\theta_{1}\right) \operatorname{tg} \phi}\right\} \\
& \left.+2 \cos ^{2} \psi \cos \left(\theta_{1}+\psi\right)\left\{\sin \left(\theta_{1}-\psi\right)-\sin \left(\theta_{2}-\psi\right) \mathrm{e}^{\left(\theta_{1}-\theta_{2}\right) \operatorname{tg} \psi}\right\}\right] .
\end{aligned}
$$




$$
\begin{aligned}
& \text { B. } \theta_{2}-\theta_{1}=\pi, \text { i.e., } \theta_{1}=\pi / 2-\psi, \\
& 1+\chi^{\prime}=\mu^{\prime}=\sin \psi \cos \psi \operatorname{tg} \phi, \\
& \chi^{\prime \prime}=\mu^{\prime \prime}=\cos ^{2} \psi \operatorname{tg} \phi .
\end{aligned}
$$

Some typical shapes of the $b-h$ loops and $\chi^{\prime}-\chi^{\prime \prime}$ curves are presented in figs. $4 \mathrm{~d}$ and 8 , respectively.

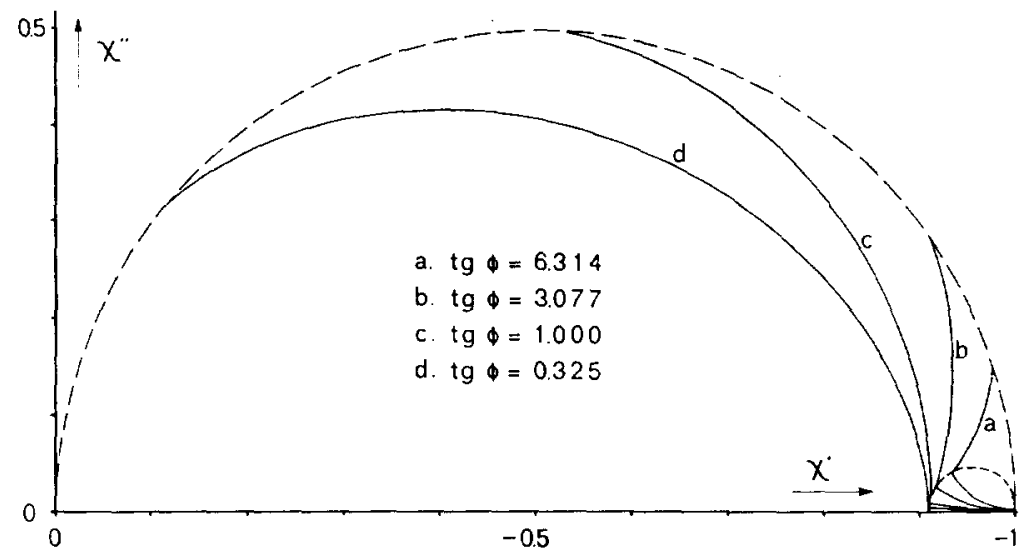

Fig. 8. $\chi^{\prime}$ vs. $\chi^{\prime \prime}$ curves according to (5.5) (between the dashed half-circles) and (5.6) (inside the smaller half circle); $\tau / \tau_{1}=10$.

The main advantage of this 3-parameter model is the fact that the $\chi^{\prime}-\chi^{\prime \prime}$ curves consist of two parts in agreement with the experimental results. Apart from the frequency only the external field dependence of the parameters $\sigma$ and $\tau / \tau_{1}$ plays a rôle. Also the slope $\mathrm{d} \chi^{\prime \prime} / \mathrm{d} \chi^{\prime}$ at $\chi=-1$ has a finite value in agreement with the experiments.

Acknowledgements. The authors are indebted to Professor H. G. de Winter for his interest in this work and for valuable discussions. They wish to thank Mr. H. A. Steffens, Mr. J. A. Ulfman and Mr. M. Caspari for their technical support. The cooperation of the university computer centre "Rekencentrum" during the calculations and in preparation of some figures is gratefully acknowledged.

\section{REFERENCES}

1) Van den Broek, J., Van der Marel, L. C. and Gorter, C. J., Physica 25 (1959) 371.

2) Bean, C. P., De Blois, R. W. and Nesbitt, L. B., J. appl. Phys. 30 (1959) 1976.

3) Cotti, P., Phys. Letters 4 (1963) 114; Phys. Kondens. Materie 3 (1964) 40.

4) Clark, A. F., Deason, V. A. and Powell, R. L., Cryogenics 12 (1972) 35.

5) De Haas, W. J. and Du Pré, F. K., Physica 5 (1938) 501.

6) Van Vleck, J. H., Phys. Rev. 59 (1941) 724 and 730.

7) Gorter, C. J., Van der Marel, L. C. and Bölger, B., Physica 21 (1955) 103. 
8) Van den Broek, J., Van der Marel, L. C. and Gorter, C. J., Physica 27 (1961) 661.

Van den Broek, J. and Van der Marel, L. C., Physica 29 (1963) 948.

9) Zenneck, J., Ann. Phys. 9 (1902) 497.

10) Wwedensky, B., Ann. Phys. 64 (1921) 609.

11) Kouwenhoven, W. B. and Daiger, G. P., Rev. sci. Instr. 5 (1934) 94.

12) Van den Berg, G. J. and Van der Marel, L. C., Suppl. Bull. I.I.F. Annexe 1955-2, 161.

13) Bean, C. P., Phys. Rev. Letters 8 (1962) 250.

14) London, H., Phys. Letters 6 (1963) 162.

15) Van der Klein, C. A. M., Elen, J. D., Wolf, R. and De Klerk, D., Physica 49 (1970) 98.

16) De Klerk, D. and Van der Klein, C. A. M., J. low Temp. Phys. 6 (1972) 1.

17) Fink, H. J., Phys. Rev. Letters 16 (1966) 447.

18) Rollins, R. W. and Silcox, J., Phys. Rev. 155 (1967) 404.

19) Casimir, H. B. G. and Du Pré, F. K., Physica 5 (1938) 507.

20) Van de Klundert, L. J. M., Induced charge transport in niobium and tin at low temperatures, Thesis, Twente University of Technology, june 1972.

21) Van de Klundert, L. J. M., Van de Braak, H. P. and Visser, D., to be published.

22) Kubo, R. and Nagamiya, T., Solid State Physics, Mc Graw-Hill (New York, 1969).

23) Van de Klundert, L. J. M., Nijnuis, H. T., Gijsbertse, E. A. and Van der Marel, L. C., to be published in Physica. 